\title{
BMJ Open Is the link between socioeconomic status and resilience mediated by reserve-building activities: mediation analysis of web-based cross-sectional data from chronic medical illness patient panels
}

Carolyn Emily Schwartz, ${ }^{\oplus 1,2}$ Jie Zhang, ${ }^{1}$ Brian Dale Stucky, ${ }^{3}$ Wesley Michael, ${ }^{4}$ Bruce David Rapkin ${ }^{5}$

To cite: Schwartz CE, Zhang J, Stucky BD, et al. Is the link between socioeconomic status and resilience mediated by reserve-building activities: mediation analysis of webbased cross-sectional data from chronic medical illness patient panels. BMJ Open 2019;9:e025602. doi:10.1136/ bmjopen-2018-025602

- Prepublication history and additional material for this paper are available online. To view these files, please visit the journal online (http://dx.doi. org/10.1136/bmjopen-2018025602).

Received 26 July 2018 Revised 6 February 2019 Accepted 17 April 2019
D) Check for updates

(c) Author(s) (or their employer(s)) 2019. Re-use permitted under CC BY-NC. No commercial re-use. See rights and permissions. Published by BMJ.

For numbered affiliations see end of article.

\section{Correspondence to} Dr Carolyn Emily Schwartz; carolyn.schwartz@deltaquest. org

\section{ABSTRACT}

Objectives The purpose of this study is to test the hypothesis that the link between socioeconomic status (SES) and resilience is mediated by reserve-building activities.

Design Cross-sectional observational study. Structural equation modelling (SEM) was used to test the mediation hypothesis.

Setting Web-based survey.

Participants Participants with a chronic medical condition were recruited from Rare Patient Voice.

Primary and secondary outcome measures DeltaQuest Reserve-Building Measure; demographic variables to capture SES; Centers for Disease Control Healthy Days Core Module; Self-Administered Comorbidity Questionnaire. Resilience was operationalised using residual modelling.

Results The study sample included 442 patients (mean age $49,85 \%$ female). SES was modelled as a bifactor model composed of general SES and specific factors for personal finance and parent's education. A series of simple mediation models predicting resilience led to the selection of three reserve-building activities for subsequent SEM-based mediation models: Active in the World, Outdoor and Exercise. The full SEM model supported the hypothesis that the relationships from both general SES and personal finance to resilience were mediated by engaging in the three reserve-building activities. In addition, the number of comorbidities partially mediated the relationship between personal finance and reserve-building. Those with more comorbidities generally had lower levels of resilience.

Conclusions This study provides suggestive evidence that reserve-building activities may be one pathway by which SES is associated with resilience: people of higher SES are more likely to engage in reserve-building activities that are intellectually stimulating, involve Outdoor pursuits and include physical Exercise. These reserve-building activities are not costly to pursue. These findings may empower patients to introduce more such reserve-building activities into their lives.
Strengths and limitations of this study

- The study uses a more comprehensive measure of reserve-building activities than previously used in past research.

- The study sample is robust $(n=442$ people with chronic illness), enabling the use of structural equation modelling to test the following mediation hypothesis: reserve-building activities mediate the relationship between socioeconomic status (SES) and resilience.

- While the sample is heterogeneous in its illness representation, it is predominantly composed of middle-aged white females who are married or living with family members.

- The data are also cross-sectional, limiting our ability to test mediation effects with certainty or causal effects.

- There are potential confounding factors, such as structural environmental factors, that were not addressed in the analysis. For example, while it may be true that many of the reserve-building activities are free or inexpensive, that does not mean that they are equally accessible across the SES continuum.

\section{INTRODUCTION}

Substantial health disparities exist in the USA, despite $18 \%$ of the gross national product expenditures being on healthcare. ${ }^{1}$ A large literature has documented the relationship between socioeconomic status (SES) on morbidity and mortality, ${ }^{23}$ and has shown that this relationship does not simply reflect the effects of poverty. ${ }^{4}$ This SES-morbidity relationship is similar across income groups, extending beyond poverty to explain relative differences in health among higher SES groups as well. ${ }^{24}$ The SES-health connection may relate to patterns of social behaviour and 
interpersonal experiences that either promote disease or protect against it. ${ }^{45}$

Recent work on the concept of reserve and reservebuilding activities may provide a useful mechanism by which (high) SES buffers health. Building on observational work in patient populations with neurological disease, a substantial body of research has documented that past and current engagement in activities that stimulate the brain may buffer against disease progression in people with multiple sclerosis, Alzheimer's disease or other neurological conditions ${ }^{6-8}$ and may be protective against cognitive impairment after chemotherapy in patients with cancer. ${ }^{9}$ The past and current 'reservebuilding activities ${ }^{10} 11$ may reflect whole-brain stimulation (eg, education, childhood enrichment activities) and 'multiple intelligences' (ie, skills ranging across intellectual, artistic and physical pursuits). ${ }^{12}$ Thus, engaging in reserve-building activities may promote better health outcomes across the health-illness spectrum (ie, resilience) by helping the individual to stimulate the brain; to remain flexible; and with higher plasticity. ${ }^{10-16}$ This flexibility may translate to more adaptive ways of coping, ${ }^{17}$ as well as to appraisal processes that emphasise the positive and focus on aspects of their life that are more controllable. ${ }^{18}$

To date, the fields of social determinants of health and reserve have remained distinct. Their interconnection led to the present study involving a heterogeneous cohort of people with chronic illness. We seek to test the hypothesis that the SES-resilience link is mediated by reservebuilding activities.

\section{METHODS \\ Sample}

Eligible participants were patients with a chronic medical condition of age 18 years or older, and able to complete an online questionnaire. Participants were recruited from Rare Patient Voice and WhatNext panels, which include people with diagnoses of a heterogeneous grouping of chronic health conditions (Rare Patient Voice) and cancer (WhatNext). The panel participants were recruited in-person at conferences and gatherings of disease-specific organisations.

\section{Patient and public involvement}

The present study did not directly involve patients or the public in its design. It did, however, follow up on a decadelong evolution. The concept of reserve-building evolved over a decade of research with chronically ill patients. The measure of reserve-building was developed over a period of 7 years, involving patient feedback and input on item development, and cognitive interviews of earlier versions of the measure. Finally, the research question 'Is reservebuilding only available to patients with substantial financial and other socioeconomic resources?' was formulated by patients in open question-and-answer sessions directed at the first author at conference presentations. Results of this research path have been provided to study participants in the form of lay-language slide presentations supplied by the first author.

\section{Procedure and design}

A web-based survey was administered in Spring 2016 using the Health Insurance Portability and Accountability Act of 1996 (HIPAA-compliant), secure SurveyGizmo engine (www.surveygizmo.com). Email invitations were sent to panel members using their standard protocol for notifying panel participants of study opportunities. No financial or other incentives were offered to participants. We followed study procedures described by Dillman's Tailored Design Method. ${ }^{19}$ The survey began with an informed consent form that participants endorsed prior to completing the questionnaires.

\section{Measures}

Reserve-building was measured with subscales from the validated DeltaQuest Reserve-Building Measure ${ }^{20}$ assessing current reserve-building activities, past reservebuilding activities and person characteristics (see ref 10 for full details of reliability and validity of the measure). The present study included the nine current-reserve-building activities subscales: Active in the World (eg, attending lectures; three items), games (eg, puzzles; three items), Outdoors (eg, spending time Outdoors; three items), creative (eg, hobbies involving working with one's hands; four items), religious/spiritual (eg, individual or group religious; three items), Exercise (eg, mild, moderate and strenuous Exercise; four items), inner life (eg, reading; three items), shopping/cooking (eg, cooking as a hobby; two items), and passive media consumption (eg, watching television; three items). Person factors related to reservebuilding included subscales for perseverance (five items), work value (two items), and current social support (five items) (see ref 20 for psychometric details).

To capture SES-related variables, we included the following variables from the DeltaQuest Reserve-Building measure's past reserve-building subscales: self and parent education, income and occupational complexity. Occupational complexity was assessed using a series of skip-logic questions querying the job that was closest to the respondent's current or past occupation which were then scored for complexity using the O*NET system. ${ }^{21}$ Additionally, we included an item asking about the respondent's difficulty paying bills. ${ }^{22}$ This item was reverse coded so that higher scores reflected lower difficulty paying bills.

Resilience was measured using a residual model approach described below in Statistical Analysis. Items from the Centers for Disease Control (CDC) Healthy Days Core Module ${ }^{23}$ was used to create the resilience score, consistent with prior work done by members of our group. ${ }^{24}$ Two items ask the respondent to indicate how many days of the past 30 days their physical (physical health problems) or mental (mental health problems) health, respectively, was not good. A third item (activities of daily living impaired (ADL impaired)) asks how 
many days of the past 30 the respondent's poor physical or mental health kept them from doing their usual activities, such as self-care, work or recreation.

Respondent demographic characteristics included age, gender, cohabitation/marital status, with whom the person lives, employment status, annual household income categories and a number of comorbidities, as measured by the Self-Administered Comorbidity Questionnaire. ${ }^{25}$

\section{Statistical analysis}

To operationalise resilience, we built on a precedent for using residual modelling to study epiphenomena. ${ }^{26-28}$ We computed a regression model with the CDC Healthy Days ADL impaired as the dependent variable, and physical health problems, mental health problems and their interaction as predictors. The residuals from the regression model were saved and multiplied by negative one $(-1)$. Thus, a high resilience score reflects fewer-than-expected days that the respondent is unable to function due to physical or mental health problems or the synergistic effect of physical and mental health problems. ${ }^{24}$

General SES was operationalised using a bifactor methodology (eg, see refs 29 30). In a bifactor model, factor loadings on the single general factor reflect the magnitude of relationships between the variables (eg, self and parent education, income and occupational complexity) and general SES. In the bifactor context, shared content between subsets of variables is captured by a second loading on a content-specific factor. For example, the residual correlation between mother's and father's education may be captured by the specific factor parent's education. Similarly, the residual correlation between income and difficulty paying bills is captured by the specific factor personal finance. In this manner, the bifactor model accounts for dependencies between items when establishing a total SES score.

Mediation models were conducted within a structural equation modelling (SEM) framework. It was hypothesised that reserve-building activities would mediate the relationship between SES and resilience. In mediation analyses, there are two types of relationships (pathways) to consider. There is the direct effect from SES to resilience, and there is the indirect effect from SES to resilience through both variables' relationship with reserve-building (the mediator; see figure 1). In this instance, mediation occurs when some (partial) or all (full) of the direct effect between SES and resilience is accounted for by the indirect effect through reserve-building.

SEM mediation analyses were conducted using Mplus V.7 software. Model fit was evaluated using the following indices: root mean square error approximation (RMSEA) $\leq 0.08$, tucker lewis index (TLI) $\geq 0.95$ and comparative fit index $(\mathrm{CFI}) \geq 0.95$. $^{31}{ }^{32}$ Analyses of observed variables were conducted using Stata V. $15^{33}$ and SPSS V.24. ${ }^{34}$ Pearson correlations were evaluated using Cohen's criteria for delineating small $(0.10<\mathrm{r}<0.30)$, medium $(0.30<\mathrm{r}<0.50)$ and large $(r>0.50)$ effect sizes,

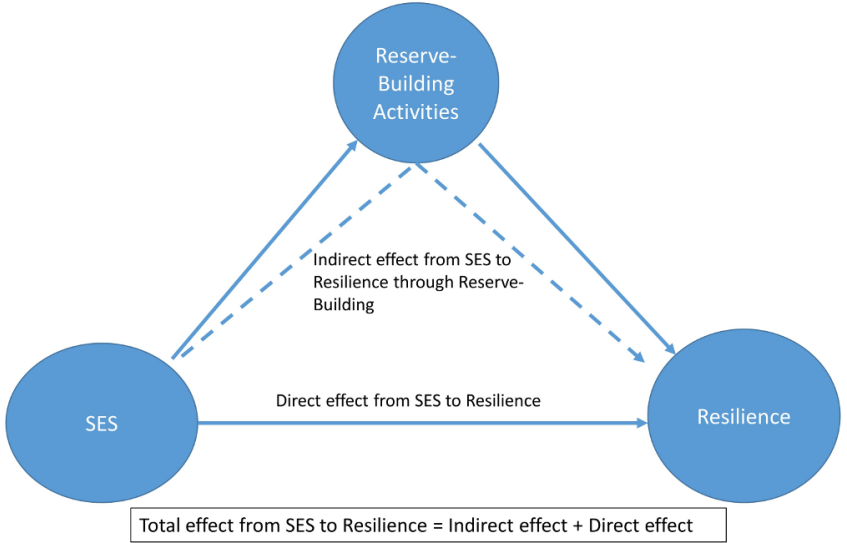

Figure 1 Theoretical model being tested. It was hypothesised that reserve-building activities would mediate the relationship between SES and resilience. Thus, the direct effect from SES to resilience would be partially or fully attenuated through both variables' relationship with reservebuilding (the mediator). SES, socioeconomic status.

hereafter referred to as small, medium and large correlations. ${ }^{35}$ We examined distributions of all variables to be included in the analysis.

\section{RESULTS}

\section{Sample}

The study sample included 442 patients. Table 1 provides the sociodemographic characteristics, international statistical classification of diseases and related health problems. - 10th revision, edition 2010 (ICD-10) categories and reported comorbidities of this heterogeneous sample. The sample had a mean age of 49 , and $85 \%$ were female. Most respondents were married and living with spouse and/or family members. The sample was predominantly white $(91 \%)$, non-Hispanic ethnicity $(92 \%)$, with $5 \%$ of respondents endorsing black race and 3\% endorsing Asian, Middle-Eastern or other. The most prevalent ICD-10 index health conditions (ie, for panel membership) were diseases of the nervous system followed by neoplasms and endocrine diseases. The most prevalent comorbidities were back pain, depression and insomnia. Sixty-nine per cent of the sample reported a family income of less than US\$100000 annually, and $69 \%$ of the sample reported that it was somewhat, very or extremely difficult to pay their bills. The majority of the sample reported past or current occupations reflecting little to medium preparation needed.

Table 2 shows descriptive statistics of the reservebuilding, person factors and demographic scores used to create the SES latent variable scores. Mother's education level was positively skewed, suggesting that most participants reported relatively low levels of maternal education. Two of the current reserve-building subscales were negatively skewed (Passive Media Consumption, Inner Life), suggested that most people spent substantial amounts of time in both of these types of activities. The other variables tested in the SEM model were normally distributed. 


\begin{tabular}{|c|c|}
\hline \multicolumn{2}{|l|}{ Variable } \\
\hline $\mathrm{n}$ & 442 \\
\hline \multicolumn{2}{|l|}{ Age } \\
\hline Mean (SD) & $48.97(12.96)$ \\
\hline \multicolumn{2}{|l|}{ Gender (\%) } \\
\hline Male & 15 \\
\hline Female & 85 \\
\hline \multicolumn{2}{|l|}{ ICD-10 diagnostic category of index condition (\%) } \\
\hline Certain infectious and parasitic diseases & 1 \\
\hline Neoplasms & 25 \\
\hline $\begin{array}{l}\text { Diseases of the blood and blood-forming organs } \\
\text { and immune mechanism disorders }\end{array}$ & 5 \\
\hline Endocrine, nutritional and metabolic diseases & 6 \\
\hline Mental and behavioural disorders & 3 \\
\hline Diseases of the nervous system & 42 \\
\hline Diseases of the ear and mastoid process & 0 \\
\hline Diseases of the circulatory system & 3 \\
\hline Diseases of the respiratory system & 3 \\
\hline Diseases of the digestive system & 1 \\
\hline Diseases of the skin and subcutaneous tissue & 1 \\
\hline $\begin{array}{l}\text { Diseases of the musculoskeletal system and } \\
\text { connective tissue }\end{array}$ & 6 \\
\hline Diseases of the genitourinary system & 0 \\
\hline $\begin{array}{l}\text { Congenital malformations, deformations and } \\
\text { chromosomal abnormalities }\end{array}$ & 1 \\
\hline
\end{tabular}

Comorbidities (\%)

\begin{tabular}{lc} 
Arthritis & 38 \\
Asthma & 19 \\
Back pain & 58 \\
Cancer (now or in the past) & 31 \\
Depression & 52 \\
Diabetes & 10 \\
Heart disease & 10 \\
\hline High blood pressure & 31 \\
\hline Insomnia & 43 \\
\hline Kidney disease & 5 \\
\hline Liver disease & 3 \\
\hline Lung disease & 11 \\
Stroke & 2 \\
\hline Ulcer or stomach disease & 13 \\
Education (\%) & \\
\hline High school or less & 14 \\
\hline Some college & 38 \\
\hline College degree & 27 \\
\hline Graduate degree & 20 \\
\hline Missing & 0 \\
\hline Father's education & 14 \\
\hline Sigh school or less & 48 \\
\hline College degree & \\
\hline
\end{tabular}

Continued
Table 1 Continued

$\begin{array}{lr}\text { Graduate degree } & 11 \\ \text { Missing } & 5\end{array}$

Mother's education

High school or less 49

Some college $\quad 30$

College degree $\quad 11$

Graduate degree 8

Missing 2

Marital status (\%)

Never married $\quad 15$

Married 60

Cohabitation/domestic partnership $\quad 7$

Separated 2

Divorced 13

Widowed 2

Missing 1

Living Situation* (\%)

Spouse/partner 69

Other relative (children, sibling, parent) 44

Friend/companion 4

Pet(s) $\quad 48$

Alone 11

Other 1

Income, US\$ (\%)

$\begin{array}{lc}\text { Less than } 15000 & 11 \\ 15001-30000 & 14 \\ 30001-50000 & 17 \\ 50001-100000 & 27 \\ 100001-150000 & 13 \\ 150001-200000 & 4 \\ \text { Over 200000 } & 2 \\ \text { Missing } & 12 \\ \text { Difficulty paying bills (\%) } & \\ \text { Extremely difficult } & 28 \\ \text { Very difficult } & 16 \\ \text { Somewhat difficult } & 24 \\ \text { Slightly difficult } & 12 \\ \text { Not difficult at all } & 16 \\ \text { Missing } & 3\end{array}$

Occupational complexity

(O*NET job zone) (\%)

\begin{tabular}{lr} 
1: Little or no preparation needed & 5 \\
2: Some preparation needed & 23 \\
3: Medium preparation needed & 24 \\
4: Considerable preparation needed & 22 \\
5: Extensive preparation needed & 7 \\
Missing & 19 \\
\hline
\end{tabular}

* May add up to more than $100 \%$ because respondents were allowed to check all that apply. ICD-10, international statistical classification of diseases and related health problems. - 10th revision, edition 2010. 
Table 2 Descriptive statistics of variables used in structural equation modelling analysis

\begin{tabular}{|c|c|c|c|c|}
\hline & Obs & Median & Variable label & Skewness \\
\hline \multicolumn{5}{|l|}{$\begin{array}{l}\text { General socioeconomic } \\
\text { status }\end{array}$} \\
\hline Mother's education & 431 & 1 & $\begin{array}{l}\text { Some high school or } \\
\text { less }\end{array}$ & 1.05 \\
\hline Job complexity & 358 & 3 & $\begin{array}{l}\text { Medium preparation } \\
\text { needed }\end{array}$ & 0.03 \\
\hline \multicolumn{5}{|l|}{ Personal finance } \\
\hline Income (US\$) & 387 & 4 & $50001-100000$ & 0.12 \\
\hline \multicolumn{5}{|c|}{ Current reserve-building subscales } \\
\hline Active in the World & 439 & 49.91 & 7.65 & 0.65 \\
\hline Games & 332 & 49.60 & 6.90 & -0.10 \\
\hline Outdoor & 438 & 49.40 & 7.63 & 0.35 \\
\hline Creative & 440 & 50.03 & 7.03 & 0.47 \\
\hline Religious/spiritual & 437 & 49.97 & 7.68 & 0.45 \\
\hline Exercise & 435 & 49.42 & 7.29 & 0.60 \\
\hline $\begin{array}{l}\text { Passive media } \\
\text { consumption }\end{array}$ & 434 & 50.27 & 6.75 & -1.25 \\
\hline
\end{tabular}

*GED test that certifies that the individual has achieved an educational level equivalent to high-school diploma.

Bold values indicate notable skewness.

Table 3 shows Pearson correlation coefficients among the variables considered in the current study. It revealed that the socioeconomic indices generally had small correlations, although mother's and father's education had moderate correlations, and difficulty paying bills had a moderate correlation with number of comorbidities. The reserve-building subscales generally had small or negligible correlations with socioeconomic indices, and small to moderate intercorrelations. The reserve-building measures generally had small or negligible correlations with the resilience score. Four reserve-building scores had small correlations with resilience: Active in the World, Outdoors, Exercise and Shopping/Cooking. The resilience score was most driven by (fewer) number of days with physical health problems. Resilience had a moderate negative correlation with number of comorbidities and difficulty paying bills, and small positive correlations with respondent's education and income tertile. Resilience was not correlated with mother's education, father's education or job complexity. The correlation matrix providing a starting point for parsimonious SEM model building, suggesting that the socioeconomic indicators be modelled as a latent variable(s), and considering only four of the nine reserve-building scores in the model: Active in the world, Outdoor, Exercise and shopping/ cooking.

\section{Step 1: bifactor model of SES}

We first modelled SES as a bifactor model with the general factor composed of respondent's education, father's education, mother's education, occupational complexity, income tertile, and endorsed difficulty paying bills (online supplementary figure 1). The specific (ie, orthogonal) factors in the bifactor model account for residual covariance between parent's education (mother's and father's education) and personal finance (income and difficulty paying bills). Note that the magnitude of the general factor loadings is small for those items that also 


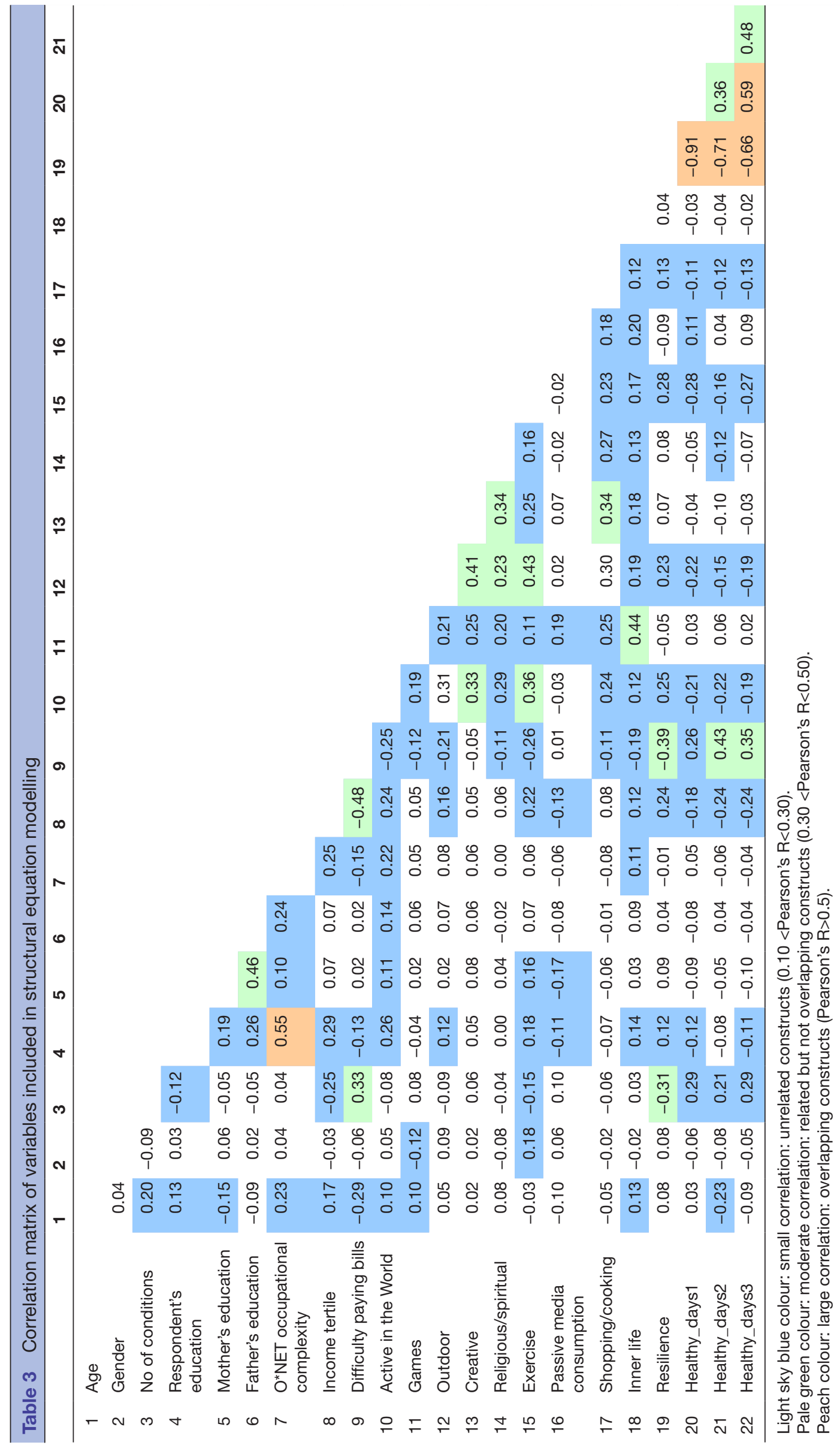


have factor loadings on parent's education and personal finance. This pattern is due to the strength of the covariance between these item subsets, and is reflected in the relatively high loadings on the specific factors. The bifactor SES model closely fit the data $\left(\chi^{2}=9.3, \mathrm{df}=7\right.$, $\mathrm{p}=0.23 ; \quad$ RMSEA $=0.027, \quad$ CFI $=0.996, \quad$ TLI $=0.992) \quad$ and provided a starting point for subsequent mediation models.

\section{Step 2: simple mediation models for reserve-building activities}

We next evaluated a series of simple mediation models where each of the nine reserve-building activities mediated the relationship between the general SES factor and Resilience (online supplementary table 1 ). The results of these models led to the selection of three reservebuilding activities for subsequent SEM-based mediation models: Active in the World, Outdoor and Exercise were significant mediators of the relationship between SES and resilience. Of note, Exercise was the strongest direct predictor for resilience.

\section{Step 3: full mediation models tested}

We then developed a mediation model where the relationship between SES (step 1) and resilience was mediated by the three reserve-building activities identified in step 2. In addition, the number of comorbidities was modelled as a covariate of resilience. The penultimate model accounted for $19 \%$ of variance in resilience but poorly fit the data $\left(\chi^{2}=229.1, \quad \mathrm{df}=36, \quad \mathrm{p}<0.01 ; \quad \mathrm{RMSEA}=0.110, \quad \mathrm{CFI}=0.796\right.$, TLI=0.689; see online supplementary table 2 ).

Review of modification indices and the correlation matrix in table 3 suggested that the penultimate mediation model was not accounting for relationships between personal finance, as defined in the bifactor model, and resilience. After adding the personal finance, latent variable as a predictor of resilience, the final mediation model accounted for $25 \%$ of the variance in resilience and closely fit the data $\left(\chi^{2}=47.4, \mathrm{df}=31, \mathrm{p}=0.03 ; \mathrm{RMSEA}=0.035\right.$, $\mathrm{CFI}=0.983$, TLI $=0.970$; see table 4 ).

As illustrated in figure 2, the relationship between resilience and both SES and personal finance is mediated by three reserve-building activities (Active in the World, Outdoor and Exercise). In other words, the bivariate relationships (SES to resilience) were significant until reserve-building activities were included in the model, and then the significant relationships were only from SES to reserve-building, but not the paths from reservebuilding to resilience. Further, the three specific reservebuilding to resilience paths have $\mathrm{p}$ values $0.06,0.12,0.17$, one of which is nearly significant. The total mediation effect considers them as a whole collection, and taken together they do account for enough of the variance to create a total mediation effect.

In addition, the personal finance to resilience path is also mediated by the participant's number of comorbid conditions. As indicated in table 4, there are significant total indirect effects from both general SES $(b=0.06$, $\mathrm{p}<0.01)$ and personal finance $(\mathrm{b}=0.13, \mathrm{p}<0.001)$ to resilience. This model supports the hypothesis that the relationship between general SES and resilience is fully mediated by engaging in the three reserve-building activities. However, there is only partial mediation from personal finance to resilience because of the remaining significant direct effect $(b=0.31, p<0.001)$. Higher levels of personal finance were also negatively associated with number of comorbidities, and those with more comorbidities generally had lower levels of resilience.

\section{Post hoc analyses}

Figure 3A-C juxtapose the relationship between reservebuilding and resilience and the mean level of each reserve-building activity, by SES-tertile group. Z-score transformations were used to compare the correlation coefficients, and analysis of variance was used to compare means by group (type I error rate of 0.05 ). Figure 3A illustrates that the relationship between Active in the World and resilience is similar across SES groups $(\mathrm{z}=0.28$, $\mathrm{p}=0.39$ ), but the mean level of these activities varied by SES group $(\mathrm{F}=7.20, \mathrm{df}=2, \mathrm{p}=0.001)$. For Outdoor activities, the associations with resilience were not statistically significant $(\mathrm{z}=1.65, \mathrm{p}<0.10)$, and the group means were not different from each other $(\mathrm{F}=1.30, \mathrm{df}=2, \mathrm{p}=0.27)$. For Exercise, however, the medium and high-SES groups showed a statistically significant difference in associations with resilience $(\mathrm{z}=2.10, \mathrm{p}<0.05)$, and the group means were not different from each other $(\mathrm{F}=2.57, \mathrm{df}=2, \mathrm{p}=0.08)$.

\section{DISCUSSION}

Our findings are consistent with the idea that the SES-resilience link is mediated by the individual's engagement in reserve-building activities. Thus, people with higher SES tend to be more resilient in the face of chronic illness by dint of their regular practice of activities that stimulate them intellectually, culturally, and physically. In other words, people with higher SES may be more likely to spend their discretionary time engaging in reserve-building activities, and this practice makes them more resilient despite their chronic illness. The post hoc analyses revealed that the association between reservebuilding and resilience was similar across SES-tertile groups for Active in the World and Outdoor activities, but not for Exercise. For Exercise, medium-SES people who engaged in more Exercise had more resilience, but this was not as strongly associated in the high-SES group. The high-SES group engaged in more active-in-the-world activities but similar levels of Outdoor and Exercise activities compared with the other SES-tertile groups. Our findings suggest that resilience is not determined by SES, but is modifiable if individuals can augment their practice of three types of reserve-building activities, such as attending lectures or concerts, participating in Outdoor activities, and engaging in physical Exercise. Choosing to engage in these three types of activities rather than other 
Table 4 Final structural equation modelling mediation model including finance as a predictor

\begin{tabular}{|c|c|c|c|c|c|}
\hline & Model & beta & se & $\mathbf{t}$ & $P$ value \\
\hline \multirow[t]{6}{*}{$\begin{array}{l}\text { Predicting reserve- } \\
\text { building }\end{array}$} & $\begin{array}{l}\text { socioeconomic } \\
\text { status (SES) } \\
\text {->Active in the } \\
\text { World }\end{array}$ & 0.33 & 0.05 & 6.48 & $<0.001$ \\
\hline & $\begin{array}{l}\text { SES ->Outdoor } \\
\text { activities }\end{array}$ & 0.14 & 0.06 & 2.47 & 0.014 \\
\hline & SES ->Exercise & 0.19 & 0.05 & 3.55 & $<0.001$ \\
\hline & $\begin{array}{l}\text { Finance ->Active in } \\
\text { the World }\end{array}$ & 0.26 & 0.05 & 4.92 & $<0.001$ \\
\hline & $\begin{array}{l}\text { Finance ->Outdoor } \\
\text { activities }\end{array}$ & 0.22 & 0.06 & 4.07 & $<0.001$ \\
\hline & Finance ->Exercise & 0.30 & 0.05 & 6.03 & $<0.001$ \\
\hline \multirow{6}{*}{$\begin{array}{l}\text { Predicting } \\
\text { resilience: direct } \\
\text { effects }\end{array}$} & SES ->resilience & 0.04 & 0.06 & 0.71 & 0.476 \\
\hline & Finance ->resilience & 0.31 & 0.07 & 4.72 & $<0.001$ \\
\hline & $\begin{array}{l}\text { Active in the World } \\
->\text { resilience }\end{array}$ & 0.08 & 0.05 & 1.57 & 0.116 \\
\hline & $\begin{array}{l}\text { Outdoor activities } \\
\text {->resilience }\end{array}$ & 0.07 & 0.05 & 1.37 & 0.170 \\
\hline & $\begin{array}{l}\text { Exercise } \\
->\text { resilience }\end{array}$ & 0.10 & 0.05 & 1.88 & 0.060 \\
\hline & $\begin{array}{l}\text { Comorbid } \\
\text { conditions } \\
\text {->resilience }\end{array}$ & -0.14 & 0.05 & -2.86 & 0.004 \\
\hline \multirow{3}{*}{$\begin{array}{l}\text { Predicting } \\
\text { resilience: total } \\
\text { indirect effects }\end{array}$} & SES ->resilience & 0.06 & 0.02 & 2.65 & 0.008 \\
\hline & Finance ->resilience & 0.13 & 0.03 & 4.06 & $<0.001$ \\
\hline & & $\mathbf{R}^{2}$ & se & $t$ & $P$ value \\
\hline \multirow[t]{11}{*}{ R square } & Education & 0.78 & 0.11 & 6.87 & $<0.001$ \\
\hline & Father education & 0.59 & 0.04 & 13.71 & $<0.001$ \\
\hline & Mother education & 0.55 & 0.04 & 13.00 & $<0.001$ \\
\hline & O*NET & 0.46 & 0.08 & 6.14 & $<0.001$ \\
\hline & Income & 0.63 & 0.04 & 15.11 & $<0.001$ \\
\hline & $\begin{array}{l}\text { Difficulty paying } \\
\text { bills }\end{array}$ & 0.58 & 0.04 & 13.12 & $<0.001$ \\
\hline & No of conditions & 0.20 & 0.05 & 3.79 & $<0.001$ \\
\hline & Active in the World & 0.18 & 0.04 & 4.18 & $<0.001$ \\
\hline & Outdoor activities & 0.07 & 0.03 & 2.39 & 0.017 \\
\hline & Exercise & 0.13 & 0.04 & 3.64 & $<0.001$ \\
\hline & Resilience & 0.25 & 0.05 & 5.54 & $<0.001$ \\
\hline
\end{tabular}

Model fit statistics: $\chi 2=47.4, \mathrm{df}=31, \mathrm{p}=0.03 ; \mathrm{RMSEA}=0.035, \mathrm{CFI}=0.983, \mathrm{TLI}=0.970$.

Path coefficients are reflected by '->'. CFI, comparative fit index; RMSEA, root mean square error approximation; TLI, tucker lewis index.

Bold values indicate notable skewness.

more passive, less stimulating activities appears to have a beneficial association with health.

A second finding of the present study is that personal finance had a direct effect on resilience: individuals with higher income and/or lower difficulty paying bills had higher resilience scores. Personal finance also played a role in the relationship between number of comorbidities and resilience: people with more financial resources tended to have fewer comorbidities. These financial resources had a small protective effect in the context of multiple comorbidities. This finding may reflect the fact that people with fewer financial resources are less likely to treat their comorbidities. ${ }^{36}$ Untreated comorbidities would likely reduce one's ability to be resilient to health challenges. 


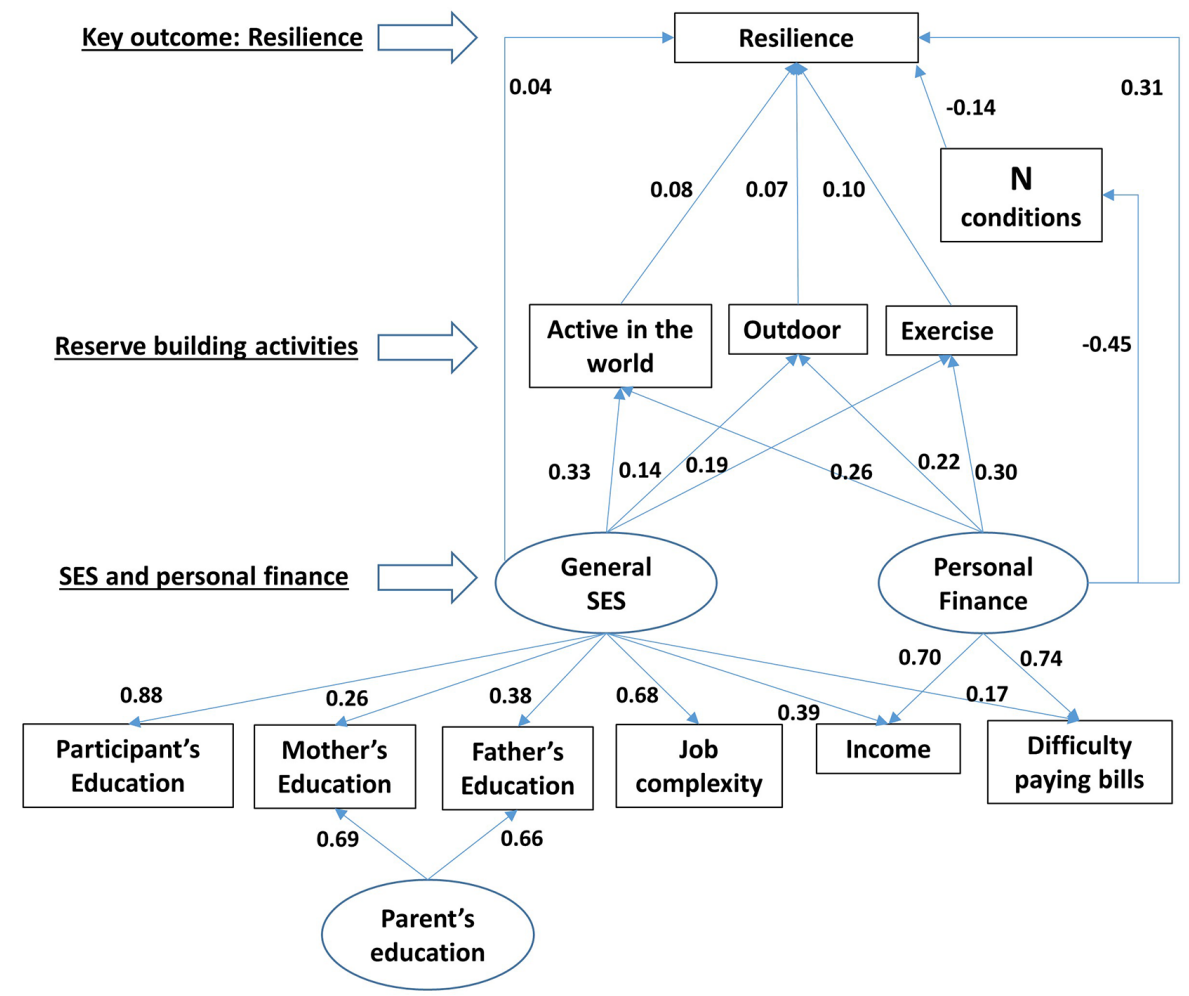

Figure 2 The full mediation model. Reserve-building activities mediate the relationships between resilience and both socioeconomic status (SES) and personal finance. The relevant reserve-building activities are Active in the World, Outdoor, and Exercise. The personal finance to resilience path is also mediated by the participant's number of comorbid conditions. As indicated in table 4 , there are significant total indirect effects from both general SES $(b=0.06, p<0.01)$ and personal finance $(b=0.13, p<0.01)$ to resilience. This model supports the hypothesis that the relationship between general SES and resilience is mediated by engaging in the three reserve-building activities. Bold values indicate significant paths $(p<0.05)$.

There has been an increasing interest in improving healthcare outcomes. In this context, 'social determinants of health' are more often than not cast in terms of deficits or negative situations that people encounter. ${ }^{37} 38$ What is missing is addressing positive social determinants of health, ${ }^{39} 40$ or person factors that confer resilience, such as reserve-building activities. Our findings suggest that it would be worthwhile to recommend the three types of activities associated with enhanced resilience. Although Exercise is standardly recommended to reduce mortality, our findings suggest that it is also beneficial for conferring day-to-day resilience in the face of physical or mental health problems. Further, other types of activities may confer similar benefits. Thus, there may be more
A

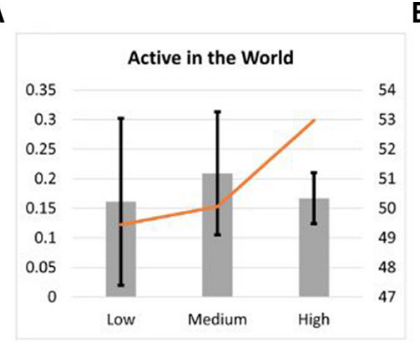

B

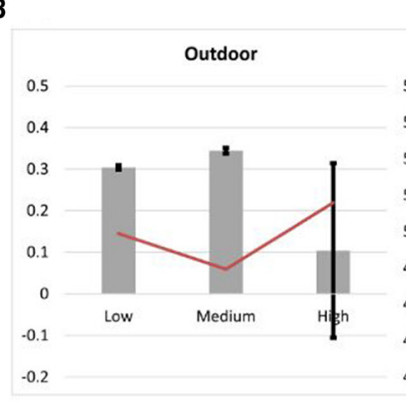

correlation coefficient -mean
C

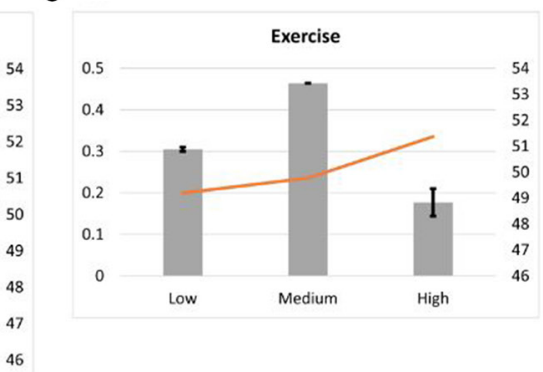

Figure 3 A-C The relationship between reserve-building and resilience. Correlation coefficients between the reserve-building activity and resilience are displayed as bar charts, with the left axis showing units. The mean values by socioeconomic status (SES) tertile are displayed as line graphs, with the right axis showing units. The relationship between Active in the World and resilience is similar across SES groups, but the mean level of these activities varied by SES group (figure 3A). For Outdoor activities, the associations with resilience and the group means were not different from each other (figure 3B). For Exercise, however, the medium and high-SES groups showed a statistically significant difference in associations with resilience, and the group means were not different from each other (figure 3C). 
options that healthcare providers can offer to people that can help them achieve better resilience and that might fit their lifestyle more easily, if Exercise is not a viable option.

The present study has a notable strength in its large and heterogeneous sample, which is useful for testing a complex hypothesis via SEM techniques. It included both income and difficulty paying bills in the operationalisation of general SES and personal finance, thus capturing complementary and distinct aspects of financial wellbeing that are often not considered in tandem. The sample had important variability in comorbidity burden, which allowed for evaluating the independent relationship between comorbidity and resilience.

The limitations of this study should, however, be noted. While the sample is heterogeneous in its illness representation, it is predominantly composed of middleaged white females who are married or living with family members. Accordingly, there was too little variation in race or ethnicity to permit inclusion of these variables in the SEM models. Thus, additional work is needed to establish relationships with resilience across age, gender, race and ethnicity groups. Indeed, age and gender were not strongly related as shown in bivariate correlations and thus were not kept in our SEM models. We are unable to quantify the response rate and thus to further identify the selection bias because we do not have access to information about who was invited to participate in the study and opted not to participate.

The data are also cross-sectional, limiting our ability to test mediation effects with certainty or causal effects. There is, however, some debate about cross-sectional mediation when the presumed mediator logically precedes the outcome, even if both are measured in the same survey. In our case, the data were collected at one time point, but the reserve-building activities logically precede the resilience score. The reserve-building items query activities done over the past 1-6 months, depending on the type of activity, and these activities are generally long-standing hobbies or practices. The SES variables are either far in the past (eg, parental education, participant education) or relatively long-standing (ie, over the past year or two, such as income or difficulty paying bills). In contrast, the resilience questions query physical and mental health sick-days of only the past 30 days. Thus, there is a logical precedence to the activities included as predictors or mediators. Nonetheless, replication of our findings in longitudinal data is needed to dispute possible 'reverse causality' (ie, the presumed mediator causes the presumed outcome or vice versa).

Finally, there are potential confounding factors, such as structural environmental factors, that were not addressed in the analysis. While it may be true that many of the reserve-building activities are free or inexpensive, that does not mean that they are equally accessible across SES groups. For example, people from lower SES backgrounds are often limited in their available free time (especially those who work more than one job) and in structural opportunities for activities like Exercise (limited availability of green space, safe places to walk/ run, access to gyms or sports facilities) and Outdoor recreation. Cooking, shopping and many cultural events also have associated costs. Even if cultural/intellectual events are free, they are often difficult to get to and people from low-SES backgrounds often have transportation barriers. Future research might address how to increase the perceived and real availability and opportunities for engaging in reserve-building activities among people with fewer SES resources and/or with more severe illness. For example, motivational interviewing techniques might be useful for addressing perceived barriers to different types of reserve-building activities (eg, cultural activities, Exercise, etc), and helping patients to find inexpensive ways to get such activities into their lives.

In summary, the present study provides suggestive evidence that reserve-building activities may be one pathway by which SES is associated with resilience: people of higher SES may be more likely to engage in reserve-building activities that are intellectually stimulating, involve Outdoor pursuits and include physical Exercise. The implications of these findings may be useful for empowering patients to introduce more such reserve-building activities in their lives, and to replace more passive activities (eg, television) with these active, salutogenic pursuits. Fundamentally, the reserve-building activities implicated in the present study are not costly to pursue ('the best things in life are free'). This critical accessibility and affordability has applications for public health interventions to enhance resilience in healthy and chronically ill individuals. It is important to ensure that individuals from low-SES backgrounds have opportunities for reserve-building activities.

\section{Author affiliations}

${ }^{1}$ DeltaQuest Foundation, Inc, Concord, California, USA

${ }^{2}$ Medicine and Orthopaedic Surgery, Tufts University School of Medicine, Boston, Massachusetts, USA

${ }^{3}$ Los Alamos National Laboratory, Santa Fe, New Mexico, USA

${ }^{4}$ Rare Patient Voice, LLC, Towson, Maryland, USA

${ }^{5}$ Epidemiology and Population Health, Albert Einstein College of Medicine, Bronx, New York, USA

Acknowledgements We are grateful to the patients who participated in this study.

Contributors Conceptualise and designed study: CES, BDS and BDR. Access to sample: WM. Data collection: CES, JZ and WM. Data Analysis: CES, JZ, BDS and BDR. Interpretation of findings: CES, JZ, BDS and BDR. Manuscript writing/editing: CES, JZ, WM, BDS and BDR.

Funding This work was unfunded by any external agency.

Competing interests None declared.

Patient consent for publication Not required.

Ethics approval The study was reviewed and approved by the New England Review Board (NEIRB\#15-254) and conforms to the principles embodied in the Declaration of Helsinki.

Provenance and peer review Not commissioned; externally peer reviewed.

Data sharing statement Participants did not consent to having their data shared with anyone other than DeltaQuest Foundation research staff.

Open access This is an open access article distributed in accordance with the Creative Commons Attribution Non Commercial (CC BY-NC 4.0) license, which permits others to distribute, remix, adapt, build upon this work non-commercially, 
and license their derivative works on different terms, provided the original work is properly cited, appropriate credit is given, any changes made indicated, and the use is non-commercial. See: http://creativecommons.org/licenses/by-nc/4.0/.

\section{REFERENCES}

1. Hartman M, Martin AB, Espinosa N, et al. National health care spending in 2016: Spending and enrollment growth slow after initial coverage expansions. Health Aff 2018;37:150-60.

2. Adler NE. Health disparities through a psychological lens. Am Psychol 2009;64:663-73.

3. Chetty R, Stepner M, Abraham S, et al. The association between income and life expectancy in the United States, 2001-2014. JAMA 2016;315:1750-66.

4. Cundiff JM, Matthews KA. Is subjective social status a unique correlate of physical health? A meta-analysis. Health Psychol 2017;36:1109-25

5. Cundiff JM, Smith TW. Social status, everyday interpersonal processes, and coronary heart disease: A social psychophysiological view. Soc Personal Psychol Compass 2017;11:e12310.

6. Schwartz CE, Quaranto BR, Healy BC, et al. Cognitive reserve and symptom experience in multiple sclerosis: a buffer to disability progression over time? Arch Phys Med Rehabil 2013;94:1971-81.

7. Stern Y, Gurland B, Tatemichi TK, et al. Influence of education and occupation on the incidence of Alzheimer's disease. JAMA 1994:271:1004-10

8. Perneczky R, Drzezga A, Boecker $\mathrm{H}$, et al. Activities of daily living, cerebral glucose metabolism, and cognitive reserve in Lewy body and Parkinson's disease. Dement Geriatr Cogn Disord 2008;26:475-81.

9. Ahles TA, Saykin AJ, McDonald BC, et al. Longitudinal assessment of cognitive changes associated with adjuvant treatment for breast cancer: impact of age and cognitive reserve. J Clin Oncol 2010;28:4434-40.

10. Schwartz CE, Rapkin BD, Healy BC. Reserve and Reserve-building activities research: key challenges and future directions. $B M C$ Neurosci 2016;17:62.

11. Schwartz CE, Snook EM, Quaranto BR, et al. Cognitive reserve and patient-reported outcomes. Multiple Sclerosis Journal 2013;19:87-105

12. Gardner H. Multiple intelligences. New horizons: Basic books, 2006.

13. Aguiar AS, Castro AA, Moreira EL, et al. Short bouts of mild-intensity physical exercise improve spatial learning and memory in aging rats: involvement of hippocampal plasticity via AKT, CREB and BDNF signaling. Mech Ageing Dev 2011;132(11-12):560-7.

14. Barulli D, Stern Y. Efficiency, capacity, compensation, maintenance, plasticity: emerging concepts in cognitive reserve. Trends Cogn Sci 2013;17:502-9.

15. Cotman CW, Berchtold NC. Exercise: a behavioral intervention to enhance brain health and plasticity. Trends Neurosci 2002;25:295-301.

16. Lövdén M, Wenger E, Mårtensson J, et al. Structural brain plasticity in adult learning and development. Neurosci Biobehav Rev 2013;37:2296-310.

17. Schwartz CE, Peng CK, Lester N, et al. Self-reported coping behavior in health and disease: assessment with a card sort game. Behav Med 1998;24:41-4.

18. Schwartz CE, Snook E, Quaranto B, et al. Cognitive reserve and appraisal in multiple sclerosis. Mult Scler Relat Disord 2013;2:36-44.

19. Dillman DA, Smyth JD, Internet CLM. phone, mail, and mixed-mode surveys: The Tailored Design Method. Fourth ed. Hoboken, NJ: John Wiley \& Sons, Inc, 2014
20. Schwartz CE, Michael W, Zhang J, et al. Assessing reserve-building pursuits and person characteristics: psychometric validation of the Reserve-Building Measure. Qual Life Res 2018;27:423-36.

21. Tsacoumis S, Willison S. O*NET Analyst Occupational Skill Ratings: Procedures: In. Organization HRR, ed. Raleigh, NC: National Center for O*NET Development, 2010.

22. Hanmer J, Cherepanov D. A single question about a respondent's perceived financial ability to pay monthly bills explains more variance in health utility scores than absolute income and assets questions. Qual Life Res 2016;25:2233-7.

23. Measuring healthy days: Population assessment of health-related quality of life. Atlanta, GA: Centers for Disease Control and Prevention, 2000.

24. Schwartz CE, Michael W, Rapkin BD. Resilience to health challenges is related to different ways of thinking: mediators of physical and emotional quality of life in a heterogeneous rare-disease cohort. Qual Life Res 2017;26:3075-88.

25. Sangha $\mathrm{O}$, Stucki $\mathrm{G}$, Liang $\mathrm{MH}$, et al. The self-administered comorbidity questionnaire: A new method to assess comorbidity for clinical and health services research. Arthritis Rheum 2003;49:156-63.

26. Reed BR, Mungas D, Farias ST, et al. Measuring cognitive reserve based on the decomposition of episodic memory variance. Brain 2010;133(Pt 8):2196-209.

27. Rapkin BD, Schwartz CE. Toward a theoretical model of quality-oflife appraisal: Implications of findings from studies of response shift. Health Qual Life Outcomes 2004;2:14.

28. Bochner B, Schwartz CE, Garcia I, et al. Understanding the impact of radical cystectomy and urinary diversion in patients with bladder cancer: treatment outcomes clarified by appraisal process. Quality of Life Research 2017;26:6.

29. Holzinger KJ, Swineford F. The Bi-factor method. Psychometrika 1937;2:41-54.

30. Cai L, Yang JS, Hansen M. Generalized full-information item bifactor analysis. Psychol Methods 2011;16:221-48.

31. Lt H, Bentler PM. Cutoff criteria for fit indexes in covariance structure analysis: Conventional criteria versus new alternatives. Structural Equation Modeling-a Multidisciplinary Journal 1999;6:1-55.

32. Browne MW, Cudeck R. Alternative ways of assesing model fit. In: Bollen KA, Long JS, eds. Testing structural equation models. Newbury Park, CA: Sage Publications, 1993.

33. Stata Statistical Software: Release 15 [program]. College Station, TX: StataCorp LLC, 2017.

34. IBM SPSS Statistics for Windows [program]. 24.0 version. Armonk, NY: IBM Corp, 2016.

35. Cohen J. A power primer. Psychol Bull 1992;112:155-9.

36. Schwartz CE, Zhang J, Michael W, et al. Reserve-building activities attenuate treatment burden in chronic illness: The mediating role of appraisal and social support. Health Psychol Open 2018;5:205510291877344

37. Capturing Social and Behavioral Domains and Measures in Electronic Health Records. 2016 http://www.nap.edu/catalog/18709/capturingsocial-and-behavioral-domains-in-electronic-health-records-phase (Accessed 21 Jun 2016)

38. Gitterman BA, Flanagan PJ, Cotton WH, et al. Poverty and child health in the United States. Pediatrics 2016:2016-339.

39. Garg A, Boynton-Jarrett R, Dworkin PH. Avoiding the Unintended Consequences of Screening for Social Determinants of Health. JAMA 2016;316:813-4

40. Adler NE, Cutler DM, Jonathan J, et al. Addressing Social Determinants of Health and Health Disparities: Discussion Paper, Vital Directions for Health and Health Care Series. Washington, DC: National Academy of Medicine, 2016. https://nam. edu/wp-content/ uploads/2016/09/addressing-social-determinantsof-health-andhealth-disparities. pdf 